... André Seidenberg, Facharzt für Allgemeinmedizin, spezialisiert auf

Drogenpatienten und Schwangerschaftsabbrüche

\title{
«Wir brauchen eine Medizinalisierung des Drogenkonsums»
}

\section{Daniel Lüthi}

Text und Bilder

Zum Thema der medizinischen Behandlung von Drogenkonsumenten siehe auch Seite 338
Zürich Platzspitz in einer kalten Winternacht. Es ist unheimlich ruhig. Wir stehen in der Dunkelheit beim Pavillon, dunkel sind auch die wenigen Gestalten hier. André Seidenberg erinnert sich gut, wie sich da, auf der Halbinsel zwischen Limmat und Sihl, einst Hunderte, ja Tausende tummelten, wie sie da hockten und Venen suchten und Drogen spritzten und diesem Land mit dem Platz hinter dem Landesmuseum in der ganzen Welt zu unrühmlicher Berühmtheit verhalfen. «Auch hier bei der HirschSkulptur lagen sie zu Dutzenden und stachen sich in die offenen Wunden», erzählt er. «Der Hirsch hatte ein rotes Auge, und die Farbe rann über seinen Kopf und Körper wie Blut.» «Needlepark» hiess der Platzspitz damals. Vor genau 20 Jahren, im Februar 1992, schlossen ihn die Behörden gewaltsam.

\section{Helfen, wo die Not ist}

Etwa 1983 versammelten sich hier die ersten Drogenabhängigen. André Seidenberg war einer der ganz wenigen Pioniere, die in diesem Milieu schon damals Nothilfe leisteten: «Wo Hilfe nötig war, gingen wir hin.» Im Laufe der Jahre wurden es Tausende von Süchtigen. «Es war ein Riesen-Basar mit allen möglichen Drogen und anderen illegalen Geschäften, rund um die Uhr, auch bei 20 Grad Celsius unter Null. Viele starben, es gab sogar Mord und Totschlag. Wir machten Medizin aus dem Koffer, beatmeten Leute mit Überdosierungen.» Und: Als einer der Ersten verteilte Seidenberg saubere Spritzen, «gegen den Willen der Behörden. Es war klar, dass wir so die Übertragung schwerer Krankheiten verhindern konnten, aber der damalige Kantonsarzt

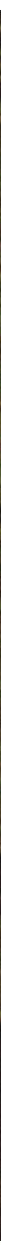


behauptete, die Abgabe von Spritzen sei verboten, und er bedrohte mich mit dem Entzug der Praxisbewilligung. Für mich wurde dies zur existentiellen Sache - man kann doch die Leute nicht einfach auf der Strasse sterben lassen.» Seidenberg kämpfte, auch für die Abgabe von Methadon.

Je grösser und je sichtbarer das Elend wurde, desto breiter wurde die öffentliche Diskussion. Und umso mehr wuchs auch die Unterstützung für Seidenberg, seine Kollegen und ihre gemeinsamen Konzepte. Aber das ganze System kollabierte, wie viele Individuen auch: «Anfang der Neunzigerjahre wurden auf dem Platzspitz etwa 6000 bis 8000 Spritzen pro Tag abgegeben, 25 Reanimationen pro Tag waren keine Seltenheit. Nichts funktionierte mehr, auch die Nothilfe nicht. Dann, im Februar 1992, wurde der Platz mit einem Eisentor abgeriegelt. Hunderte von Drogensüchtigen wurden auf die Strasse getrieben, die Bahnhofstrasse, den Sihlquai, während des Laufens versuchten einige noch, sich Spritzen zu setzen, aber Polizisten in Kampfmontur jagten diese Scharen von Elendsgestalten vor sich her wie Abfall. Später setzte sich die Szene im Letten fest. Aber auch dieses Areal wurde geräumt und geschlossen.»

\section{Die Kraft der Erfahrung}

«Der hundertjährige Drogenkrieg führt zu keiner Lösung», ist Seidenberg überzeugt, «und das Justizsystem kann nur im Nachhinein, meist ausgrenzend, tätig werden, die ständige Kriminalisierung bringt nichts. Wir brauchen eine Medizinalisierung des Drogenkonsums. Nur das Gesundheitssystem mit seiner ganzen Power, seinem wissenschaftlichen Hintergrund und all seinen Akteuren ist in der Lage, dieses Thema und seine Probleme zu bewältigen - niederschwellig und mit einem Angebot, das die Nachfrage deckt, abgestuft nach Gefahren, also evidence-based wie gehabt, und im Sinne eines Prozesses, der sich stets verändert.»

Seidenberg poltert nicht, wenn er referiert. Er spricht eindringlich, mit der Kraft der Überzeugung und der eigenen Erfahrung. Seine Glaubwürdigkeit hat also sicher vor allem damit zu tun, dass er weiss, wovon er spricht. Nicht nur aufgrund seiner ärztlichen Arbeit mit Patienten, sondern auch aufgrund von Selbstversuchen: «Schon vor dem Medizinstudium war mir klar, dass ich nicht nur lesen will, sondern auch selber erleben und erleiden. Es hat mich als junger Mensch gereizt, auch gefährliche Dinge auszuprobieren. Und so versuchte ich praktisch alle Substanzen irgendeinmal. Die Angst gehörte meist dazu. Und oft ein kötzeriges Gefühl. Die meisten Drogen führten $\mathrm{zu}$ unangenehmen Erlebnissen: Heroin zum Beispiel erlebte ich wie das Ertrinken in einer süssen Melasse - grässlich. LSD provozierte extreme körperliche Sensationen. Ketalar - ein komischer Trip. Und beim Kokain begann ich zu spüren, wie schnell es abhängig machen kann. Am schwierigsten jedoch war für mich das Aufhören beim Nikotin.» Seidenberg hält inne und schmunzelt auf

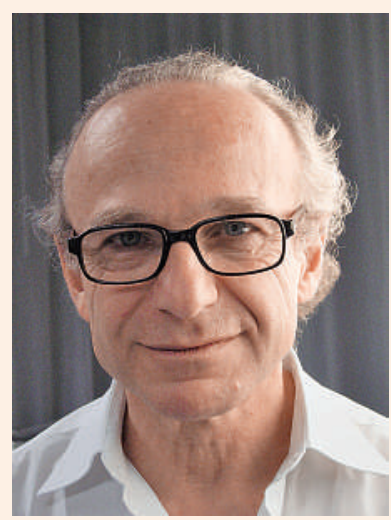

\section{André Seidenberg}

Dr. med. André Seidenberg wurde 1951 in Zürich geboren. Er wuchs im Niederdorf auf, sein Vater war Buchhändler. Eidgenössische Matura und Medizinstudium absolvierte er ebenfalls in Zürich, das Staatsexamen machte er 1978. Nach dreijähriger Assistenzzeit in der Nephrologie, Neurochirurgie und Inneren Medizin arbeitete er zusammen mit zwei anderen Ärzten und drei Zahnärzten in seiner ersten Gruppenpraxis «Plaffenwatz». Schon dort spezialisierte er sich auf die beiden Gebiete, denen er bis heute treu geblieben ist: die Behandlung von drogenkranken Menschen und den Abbruch von unerwünschten Schwangerschaften. 1985 bis 1995 führte er eine Praxis für Allgemeinmedizin in Zürich-Altstetten. In der gleichen Zeit war er als Arzt sehr aktiv in der Zürcher Drogenszene, vor allem auf dem Platzspitz. 1991 war er Mitbegründer von ARUD, der «Arbeitsgemeinschaft für einen risikoarmen Umgang mit Drogen». Sie gründete das ZOKL 1, das «Zürcher Opiat-Konsum-Lokal» und 1994 das ZOKL 2. 1993 kam es in der Schweiz unter massgeblicher Mitwirkung von André Seidenberg zu den ersten Versuchen mit kontrollierter Heroinabgabe, die weltweit für Aufsehen sorgten. Er ist Co-Autor eines medizinischen Standardwerkes zur ambulanten opioidgestützten Behandlung [1]. Seit 1997 führt Seidenberg eine Praxis für Allgemeinmedizin beim Zürcher Central. In über $\mathbf{3 0}$ Jahren als Arzt hat er rund 3000 Heroinabhängige behandelt und $\mathbf{3 0 0 0}$ Schwangerschaftsabbrüche durchgeführt. Er ist Research Fellow und Lehrbeauftragter des Instituts für Hausarztmedizin und Versorgungsforschung der Universität Zürich. André Seidenberg ist Vater von drei erwachsenen Kindern. Zusammen mit seiner Frau, der Psychologin Marianne Seidenberg-Jenny, die in seiner Praxis mitarbeitet, lebt er in Zürich.

1 Seidenberg A, Honegger U. Methadon, Heroin und andere Opioide. Bern: Hans Huber; 1998. 
seine besondere, spitzbübische Art: «Na ja, man kann auch auf Sport abfahren. Auch dies habe ich selber erlebt, beim Marathon oder Triathlon. Als Student bin ich zeitweise auf Speed, also auf Amphetamine, abgefahren, um zu arbeiten, ohne müde zu werden. Als ich aber vor 20 Jahren wegen der Drogenmisere bis zu 100 Stunden pro Woche arbeitete, brauchte ich selber keine Drogen.»

Kurz: Seidenberg hat am eigenen Leib viel erlebt; gerade damals, im wilden 68 und den paar folgenden Jahren. «Ich habe davon profitiert und meine Patienten auch. Aber ich hatte natürlich auch Glück. Im Gegensatz zu anderen, die starben. Ich bin also kein Vorbild: Man muss nicht Drogen genommen haben, um ein guter 〈Drogen-Doktor` sein zu können.»

André Seidenberg ist einer, der immer wieder an die Grenzen geht. Auch bei seinem unermüdlichen Einsatz für bessere Zeiten im Drogenbereich. Nicht immer aber mit Erfolg, wie er selber bilanziert. So sei die kontrollierte Heroinabgabe ein Tropfen auf den heissen Stein geblieben: «Damit erreicht man bloss drei Prozent der drogenkonsumierenden Population, das ist doch irrelevant. Dabei könnten die meisten normal leben, wenn ihr Nachschub gewährleistet wäre. Das hat mich schon schwer frustriert und resignieren lassen, sogar in die Depression getrieben. Aber die Schweiz kann dies nicht im Alleingang durchziehen, das sehe ich heute ja ein. So lange die USA ihren weltweiten Drogenkrieg nicht beenden, kann nichts ändern.»

\section{«Es ist eine Ecke, in der man sich leicht verdächtig macht.»}

Einen Dämpfer erlebte Seidenberg auch mit seiner Idee, Versuche mit einer staatlich kontrollierten Kokain-Abgabe zu lancieren: «Ich tüftelte an einem Inhalationsspray mit Fingerprint-Sicherung herum, ein richtiges Hightech-Tool wäre das geworden. Diese Entwicklung hätte aber Millionen gekostet, da hätte es mehr als politischen Willen gebraucht.»

Natürlich habe seine ärztliche Tätigkeit auch einen politischen Hintergrund, sagt Seidenberg: «Medizin ist ein wichtiges Instrument für den Zusammenhalt der Gesellschaft, der Gesellschaftsvertrag sieht vor, dass wir füreinander schauen, wenn wir krank und gebrechlich sind. Deshalb will ich gute Medizin gerade dort anbieten, wo sie nicht selbstverständlich ist. Da will und muss ich als Arzt hinschauen.»

\section{Leben retten - und verhindern}

André Seidenberg kümmert sich vor allem um Menschen, die es in dieser Gesellschaft schwieriger haben als andere. Und dazu gehören für ihn Frauen, die unerwünscht schwanger geworden sind, Migrantinnen beispielsweise: «Eine unerwünschte Schwangerschaft bedeutet noch heute, dass eine Biografie zerstört wird. Deshalb bringen sich Frauen gerade in Afrika oder Lateinamerika immer wieder in Lebensgefahr, indem sie mit Spülungen oder gefährlichen Instrumenten selber versuchen, den Fötus aus ihrem Körper zu entfernen. Unsafe abortion ist dort bei Frauen im fruchtbaren Alter die häufigste Todesursache.» Und in der Schweiz? «Wir haben hier die tiefste Schwangerschaftsabbruch-Rate der Welt - ein gutes Zeichen für ein fortschrittliches System. Aber dazu gehören eben niederschwellige Angebote von hoher Qualität, so auch Schwangerschaftsabbrüche in einer Allgemeinpraxis.» Drei Fragen stelle er jeder Frau, die mit diesem Wunsch zu ihm komme: «Sind Sie sicher, dass Sie diesen Abbruch wollen? Ist dies Ihre eigene Entscheidung? Und haben Sie jemanden, mit dem Sie darüber sprechen können?» Und was antwortet Seidenberg, wenn man ihm vorwirft, dass er als Arzt nicht nur Leben rettet, sondern eben auch Leben verunmöglicht, ja tötet? Er zitiert das Gesetz: «Je weiter fortgeschritten eine Schwangerschaft ist, umso dringlicher müssen die Gründe für einen Abbruch sein, und das ist gut so. Im Übrigen ist ein Embryo nur ein Teil einer schwangeren Frau, und noch kein eigenständiges Leben. Deshalb gilt es, das Leben, die Integrität der Frau zu schützen, denn sie ist ein ganzer Mensch.»

Kalt lässt ihn eine Abtreibung keineswegs: «Ich sauge ab bis 14 Wochen - das ist happig, das berührt mich, ich bin ja nicht aus Holz. Aber mit solch existentiellen Fragen sind alle konfrontiert, die in der Medizin tätig sind.» Etwas speziell seien seine Spezialitäten schon, schiebt er nach: «Ja, es ist eine Ecke, in der man sich leicht verdächtig macht. Deshalb versuche ich immer, sorgfältig und genau zu sein. Und deshalb arbeite ich mit den Daten aus der eigenen Praxis auch wissenschaftlich mit Instituten der Universität Zürich zusammen.»

Und, einmal mehr: Wichtig sei es, sagt André Seidenberg, immer auch sich selber zu reflektieren. «Es geht ja nicht darum, dass ich meine eigenen Vorstellungen von Fruchtbarkeit oder meine eigenen Probleme mit Abstinenz den Patientinnen und Patienten quasi überstülpe. Meine eigenen Geschichten muss ich für mich selber anschauen und lösen, und zwar täglich.»

\section{Die nächste «Begegnung mit ...»}

Einmal im Monat stellt die Schweizerische Ärztezeitung eine Persönlichkeit vor, die sich im Gesundheitswesen engagiert. Im März schildert Daniel Lüthi seine Begegnung mit Jonathan Spycher, Chefarzt Orthopädie am Spital Interlaken. 